\title{
Asymptotic dependence of bivariate maxima
}

\author{
Helena Ferreira \& Marta Ferreira
}

To cite this article: Helena Ferreira \& Marta Ferreira (2019) Asymptotic dependence of bivariate maxima, Communications in Statistics - Theory and Methods, 48:13, 3269-3279, DOI: 10.1080/03610926.2018.1475568

To link to this article: https://doi.org/10.1080/03610926.2018.1475568

曲 Published online: 22 Nov 2018.

Submit your article to this journal $\pi$

Џ Article views: 76

Q View related articles $₫$

View Crossmark data $\asymp$ 


\title{
Asymptotic dependence of bivariate maxima
}

\author{
Helena Ferreira ${ }^{a}$ and Marta Ferreira ${ }^{b, c, d}$ \\ aUniversidade da Beira Interior, Centro de Matemática e Aplicações (CMA-UBI), Avenida Marquês d'Avila e Bolama,

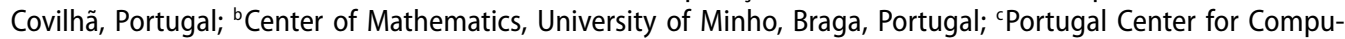

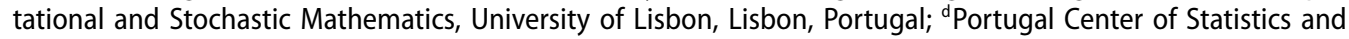 \\ Applications, University of Lisbon, Lisbon, Portugal
}

\section{ABSTRACT}

The Ledford and Tawn model for the bivariate tail incorporates a coefficient, $\eta$, as a measure of pre-asymptotic dependence between the marginals. However, in the limiting bivariate extreme value model, $G$, of suitably normalized component-wise maxima, it is just a shape parameter without reflecting any description of the dependency in $G$. Under some local dependence conditions, we consider an index that describes the pre-asymptotic dependence in this context. We analyze some particular cases considered in the literature and illustrate with examples. A small discussion on inference is presented at the end.

\section{ARTICLE HISTORY}

Received 28 September 2017 Accepted 7 May 2018

\section{KEYWORDS}

Extreme value theory; stationary sequences; asymptotic dependence; dependence conditions

2010 MATHEMATICS SUBJECT CLASSIFICATION 60G70

\section{Introduction}

Consider a stationary sequence $\left\{\left(X_{n}, Y_{n}\right)\right\}_{n \geq 1}$ with distribution function (df) $F$ belonging to the maximum domain of attraction of a bivariate extreme values (BEV) $\mathrm{df} G$. The marginals of $G, G_{X}$ and $G_{Y}$, are also extreme value df's and attract the maximum of $\left\{X_{n}\right\}$ and $\left\{Y_{n}\right\}$, respectively. The central result of the univariate extreme values theory, called Extremal Types Theorem, establishes the three possible limiting extreme value df's of the suitably normalized maximum of an independent and identically distributed (i.i.d.) sequence. This result was extended to stationary sequences under a distributional mixing condition $\mathrm{D}$ which states that the variables tend to independence as they get apart in time (Leadbetter, Lindgren, and Rootzén 1983 and references therein).

The degree of dependence between $G_{X}$ and $G_{Y}$ can be evaluated through the extremal coefficient, $\varepsilon \in[1,2]$ (Tiago de Oliveira 1962-1963; Smith 1990), such that

$$
P\left(G_{X}(X) \leq u, G_{Y}(Y) \leq u\right)=u^{\varepsilon}, u \in[0,1]
$$

assuming that the random pair $(X, Y)$ has $\mathrm{df} G$. Sufficient conditions to have $\varepsilon=2$, that is, independence between $M_{n}^{(1)}=\max _{i=1}^{n} X_{i}$ and $M_{n}^{(2)}=\max _{i=1}^{n} Y_{i}$, suitably normalized, were presented in literature, both in the case of no clustering of high values within $\left\{X_{n}\right\}$ and $\left\{Y_{n}\right\}$ (Davis 1982), as well as, in the case that such clustering is allowed (Pereira, Martins, and Ferreira 2017). This latter scenario means that extreme events tend to occur in groups. The extremal index (Leadbetter, Lindgren, and Rootzén 1983), usually denoted $\theta$, measures the tendency for data to form clusters. Whenever $\theta=1$, the extreme values tend to occur isolated and is a form of asymptotic independence. This may mean that either the data are

CONTACT Marta Ferreira msferreira@math.uminho.pt $@$ Center of Mathematics, University of Minho, Braga, Portugal; Portugal Center for Computational and Stochastic Mathematics, University of Lisbon, Lisbon, Portugal; Portugal Center of Statistics and Applications, University of Lisbon, Lisbon, Portugal. 
independent, or there is eventually a residual dependence that vanishes as $n$ tends to infinity and thus, in the limit, leading to the occurrence of isolated extremes. As far as we know, there is no discussion about this pre-asymptotic dependence in neither of these cases, i.e., the dependence between $M_{n}^{(1)}$ and $M_{n}^{(2)}$ with large $n$ in concomitance with the independence between $G_{X}$ and $G_{Y}$.

The topic of pre-asymptotic dependence, also denoted asymptotic independence, is assigned in the model of Ledford and Tawn $(1996,1997)$, in which we base our formulation of the joint right tail of $\left(X_{i}, Y_{j}\right)$. More precisely, for $\tau_{1}, \tau_{2}>0$, and denoting $f_{n} \sim g_{n}$ whenever $f_{n} / g_{n} \rightarrow a \neq 0$, as $n \rightarrow \infty$, we consider

$$
n P\left(X_{i}>\frac{n}{\tau_{1}}, Y_{j}>, \frac{n}{\tau_{2}}\right) \sim n^{-\left(1 / \eta_{i j}-1\right)} \mathcal{L}_{\eta_{i j}}\left(\frac{n}{\tau_{1}}, \frac{n}{\tau_{2}}\right)
$$

$i, j=1, \ldots, n$, where $\eta \equiv \eta_{i, j} \in(0,1]$ and $\mathcal{L} \equiv \mathcal{L}_{\eta_{i j}}$ is a slowly varying function, i.e., there exists $g$ such that, $\forall x, y>0$ and $c>0$,

$$
g(x, y)=\lim _{t \rightarrow \infty} \frac{\mathcal{L}(t x, t y)}{\mathcal{L}(t, t)} \text { and } g(c x, c y)=g(x, y)
$$

We have asymptotic independence if $\eta<1$ or if $\eta=1$ and $\mathcal{L}\left(\frac{n}{\tau_{1}}, \frac{n}{\tau_{2}}\right) \rightarrow 0$, as $n \rightarrow \infty$, and tail dependence if $\eta=1$ and $\mathcal{L}\left(\frac{n}{\tau_{1}}, \frac{n}{\tau_{2}}\right) \rightarrow a>0$. The variables $X_{i}$ and $Y_{j}$ are (almost) independent if $\eta=1 / 2$ and positively and negatively associated whenever $\eta>1 / 2$ and $\eta<1 / 2$, respectively. Ledford and Tawn (1996) showed that problems arise in modeling and inference if a pre-asymptotic dependence takes place and is ignored. See also Bortot and Tawn (1998) and Poon, Rockinger, and Tawn (2003).

Suppose, without loss of generalization, that $F$ has standard Fréchet marginals $F_{X}$ and $F_{Y}$, and thus also $G_{X}$ and $G_{Y}$. The Ledford and Tawn (Ledford and Tawn 1996, 1997) model assumption for the bivariate tail of $\mathrm{G}$, which is given by

$$
\bar{G}(u, u)=1-2 u+u^{\varepsilon}=(1-u)(2-\varepsilon)+(1-u)^{2} \varepsilon(\varepsilon-1) / 2+o\left((1-u)^{2}\right) \text {, as } u \uparrow 1
$$

would take us to $\eta=1 / 2$ when $\varepsilon=2$. Therefore, in this case, $\eta$ cannot be interpreted as a pre-asymptotic dependence coefficient as in other df's which are not BEV. On the other hand, the Ledford and Tawn assumption to model the tail of $F$, although it allows interpreting $\eta$ as a coefficient of pre-asymptotic dependence between the marginals $F_{X}$ and $F_{Y}$, it appears in $G$, after suitable normalization of $M_{n}^{(1)}$ and $M_{n}^{(2)}$, as a shape parameter (Ramos and Ledford 2011) without expression in the description of the dependence of $G$.

Here, we discuss the conditions about the modeling in Equation (1) that will lead to dependence between the marginals of $G$ or to independence, describing in this case the type of pre-asymptotic dependence. On the local behavior of each marginal sequence $\left\{X_{n}\right\}$ and $\left\{Y_{n}\right\}$, we will assume that they satisfy Chernick, Hsing, and McCormick (1991) conditions, $D^{(s)}\left(u_{n}\right)$ and $D^{(t)}\left(v_{n}\right)$, for some $s \geq 1$ and $t \geq 1$, allowing clusters of extremes separated at least $s$ and $t$, respectively, and together satisfy a local condition $D^{(k)}\left(u_{n}, v_{n}\right)$ regulating the joint location of clusters. A new index encompassing all types of asymptotic dependence between $M_{n}^{(1)}$ and $M_{n}^{(2)}$ will be presented in Section 2. In Section 3 we analyze the possible forms of pre-asymptotic dependence between $M_{n}^{(1)}$ and $M_{n}^{(2)}$ on some particular cases considered in the literature, along with illustrative examples. A discussion on Section 4 gives some insight about possible inference in this framework. 


\section{Index of asymptotic dependence between $M_{n}^{(1)}$ and $M_{n}^{(2)}$}

Consider $\left\{\left(X_{n}, Y_{n}\right)\right\}$ a stationary sequence with standard Fréchet marginals and, for $\left\{\left(u_{n}, v_{n}\right)\right\}$ such that $n\left(1-F_{X}\left(u_{n}\right)\right) \rightarrow \tau_{1}>0$ and $n\left(1-F_{Y}\left(v_{n}\right)\right) \rightarrow \tau_{2}>0$, as $n \rightarrow \infty$, it is valid the condition $D\left(u_{n}, v_{n}\right)$ of Hsing (1989), meaning that $\alpha_{n, l_{n}} \rightarrow 0$ for some $l_{n}=o(n)$, as $n \rightarrow \infty$, where

$$
\begin{aligned}
\alpha_{n, l}= & \max \left\{\left|P\left(\max _{i \in A} X_{i} \leq u_{n}, \max _{i \in B} Y_{i} \leq v_{n}\right)-P\left(\max _{i \in A} X_{i} \leq u_{n}\right) P\left(\max _{i \in B} Y_{i} \leq v_{n}\right)\right|\right. \\
& : A \subset\{1,2, \ldots, j\}, B \subset\{j+l, j+l+1, \ldots, n\}, 1 \leq j \leq n-l\} \\
& n \geq 1,1 \leq l \leq n-1
\end{aligned}
$$

Condition $D\left(u_{n}, v_{n}\right)$ extends the univariate distributional mixing condition $D$ in Leadbetter, Lindgren, and Rootzén (1983) to the bivariate case and thus also allows to extend the Extremal Types Theorem to a stationary sequence of random vectors Hsing (1989).

Furthermore, regarding the local behavior of each marginal sequence, we assume that $\left\{X_{n}\right\}$ satisfies the Chernick, Hsing, and McCormick (1991) dependence condition $D^{(s)}\left(u_{n}\right)$, for some $s \geq 1$, i.e.,

$$
n P\left(X_{1}>u_{n}, M_{2, s}^{(1)} \leq u_{n}<M_{s+1, r_{n}}^{(1)}\right) \rightarrow 0, \text { as } n \rightarrow \infty
$$

where $M_{i, j}^{(1)}=\max _{l \in\{i, \ldots, j\}} X_{l}$ with $\max _{l \in\{i, \ldots, j\}} X_{l}=-\infty$ if $i>j$ and $r_{n}=\left[n / k_{n}\right]$ for some $\left\{k_{n}\right\}$ such that

$$
k_{n} l_{n} / n \rightarrow 0, k_{n} / n \rightarrow 0, k_{n} \alpha_{n, l_{n}} \rightarrow 0
$$

Likewise we use notation $M_{i, j}^{(2)}=\max _{l \in\{i, \ldots, j\}} Y_{l}$, with $\max _{l \in\{i, \ldots, j\}} Y_{l}=-\infty$ if $i>j .\left\{Y_{n}\right\}$ satisfies $D^{(t)}\left(v_{n}\right)$, for some $t \geq 1$, with the same sequence $\left\{k_{n}\right\}$, without loss of generality. Both conditions allow clusters of exceedances of $u_{n}$ and $v_{n}$, for $\left\{X_{n}\right\}$ and $\left\{Y_{n}\right\}$, respectively, separated at least $s \geq 1$ and $t \geq 1$. Concerning the joint location of the clusters of $\left\{X_{n}\right\}$ and $\left\{Y_{n}\right\}$, we admit that they are distant from each other at most $k \geq 0$, i.e.,

$$
k_{n} \sum_{\substack{i=1 \\|i-j|>k}}^{r_{n}} \sum_{\substack{j=1 \\ r_{n}}}\left(X_{i}>u_{n}, M_{i+1, i+s-1}^{(1)} \leq u_{n}, Y_{j}>v_{n}, M_{j+1, j+t-1}^{(2)} \leq v_{n}\right) \rightarrow 0, \text { as } n \rightarrow \infty
$$

This condition will be denoted $D^{(k)}\left(u_{n}, v_{n}\right)$ and simplifies the description of the dependence between $G_{X}$ and $G_{Y}$ through the asymptotic behavior of the joint tail of $X_{i}$ and $Y_{j}$ for a finite number of pairs $(i, j)$. Observe that the simpler statement

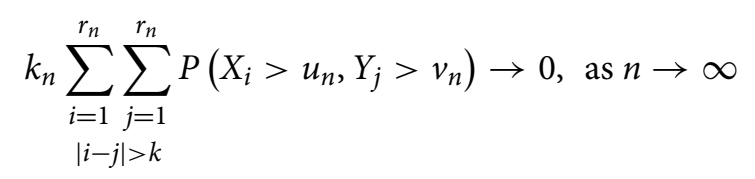

implies $D^{(k)}\left(u_{n}, v_{n}\right)$ in Equation (6) and thus can be used for checking the validity of this latter.

Lemma 2.1. If $\left\{\left(X_{n}, Y_{n}\right)\right\}$ satisfies condition $D\left(u_{n}, v_{n}\right)$ in Equation (3) for coefficients $\left\{\alpha_{n}, l_{n}\right\}$, $\left\{X_{n}\right\}$ satisfies $D^{(s)}\left(u_{n}\right),\left\{Y_{n}\right\}$ satisfies $D^{(t)}\left(v_{n}\right)$ and $\left\{\left(X_{n}, Y_{n}\right)\right\}$ satisfies $D^{(k)}\left(u_{n}, v_{n}\right)$ for some $\left\{k_{n}\right\}$ 
satisfying Equation (5), then

$$
\begin{aligned}
& \lim _{n \rightarrow \infty} P\left(M_{n}^{(1)} \leq u_{n}, M_{n}^{(2)} \leq v_{n}\right) \\
= & \exp \left\{-\lim _{n \rightarrow \infty} n P\left(X_{1}>u_{n} \geq M_{2, s}^{(1)}\right)-n P\left(Y_{1}>v_{n} \geq M_{2, t}^{(2)}\right)\right. \\
& \left.+\lim _{n \rightarrow \infty} \sum_{j=0}^{2 k} n P\left(X_{k+1}>u_{n} \geq M_{k+2, k+s}^{(1)}, Y_{j+1}>v_{n} \geq M_{j+2, j+t}^{(2)}\right)\right\}
\end{aligned}
$$

Proof. From condition $D\left(u_{n}, v_{n}\right)$ and the stationarity assumption, we have (Hsing 1989; Lemma 4.1),

$$
\begin{aligned}
\lim _{n \rightarrow \infty} P\left(M_{n}^{(1)} \leq u_{n}, M_{n}^{(2)} \leq v_{n}\right) & =\lim _{n \rightarrow \infty} P^{k_{n}}\left(M_{r_{n}}^{(1)} \leq u_{n}, M_{r_{n}}^{(2)} \leq v_{n}\right) \\
& =\lim _{n \rightarrow \infty}\left(1-\frac{k_{n} P\left(\left\{M_{r_{n}}^{(1)}>u_{n}\right\} \cup\left\{M_{r_{n}}^{(2)}>v_{n}\right\}\right)}{k_{n}}\right)^{k_{n}} \\
& =\exp \left\{-\lim _{n \rightarrow \infty} k_{n} P\left(\left\{M_{r_{n}}^{(1)}>u_{n}\right\} \cup\left\{M_{r_{n}}^{(2)}>v_{n}\right\}\right)\right\}
\end{aligned}
$$

Under conditions $D^{(s)}\left(u_{n}\right)$ for $\left\{X_{n}\right\}$ and $D^{(t)}\left(v_{n}\right)$ for $\left\{Y_{n}\right\}$, we have that (Chernick, Hsing, and McCormick 1991; Proposition 1.1 and references therein)

$$
\lim _{n \rightarrow \infty} k_{n} P\left(M_{r_{n}}^{(1)}>u_{n}\right)=\lim _{n \rightarrow \infty} n P\left(X_{1}>u_{n}, X_{2} \leq u_{n}, \ldots, X_{s} \leq u_{n}\right)
$$

and

$$
\lim _{n \rightarrow \infty} k_{n} P\left(M_{r_{n}}^{(2)}>v_{n}\right)=\lim _{n \rightarrow \infty} n P\left(Y_{1}>v_{n}, Y_{2} \leq v_{n}, \ldots, Y_{t} \leq v_{n}\right)
$$

In what follows, we apply a commonly used extreme values technique that consists in omitting terms which summation converges to zero, as $n \rightarrow \infty$, under the validity of dependence conditions (see, e.g., Leadbetter and Nandagopalan 1989). More precisely, under $D^{(k)}\left(u_{n}, v_{n}\right)$ and the stationarity,

$$
\begin{aligned}
& \lim _{n \rightarrow \infty} k_{n} P\left(M_{r_{n}}^{(1)}>u_{n}, M_{r_{n}}^{(2)}>v_{n}\right) \\
& =\lim _{n \rightarrow \infty} k_{n} \sum_{i=1}^{r_{n}} \sum_{j=1}^{r_{n}} P\left(X_{i}>u_{n}, M_{i+1, r_{n}}^{(1)} \leq u_{n}, Y_{j}>v_{n}, M_{j+1, r_{n}}^{(2)} \leq v_{n}\right) \\
& \quad=\lim _{n \rightarrow \infty} k_{n} \sum_{i=1}^{r_{n}} \sum_{j=1}^{r_{n}} P\left(X_{i}>u_{n}, M_{i+1, r_{n}}^{(1)} \leq u_{n}, Y_{j}>v_{n}, M_{j+1, r_{n}}^{(2)} \leq v_{n}\right) \\
& \quad=\lim _{n \rightarrow \infty} k_{n} \sum_{i=1}^{r_{n}} \sum_{j=1}^{r_{n}} P\left(X_{1}>u_{n}, M_{2, r_{n}-i+1}^{(1)} \leq u_{n}, Y_{j-i+1}>v_{n}, M_{j-i+2, r_{n}-i+1}^{(2)} \leq v_{n}\right) \\
& |i-j| \leq k
\end{aligned}
$$




$$
\begin{aligned}
& =\lim _{n \rightarrow \infty} k_{n} \sum_{i=1}^{r_{n}} \sum_{j=-k}^{k} P\left(X_{1}>u_{n}, M_{2, r_{n}-i+1}^{(1)} \leq u_{n}, Y_{j+1}>v_{n}, M_{j+2, r_{n}-i+1}^{(2)} \leq v_{n}\right) \\
& =\lim _{n \rightarrow \infty} k_{n} \sum_{i=1}^{r_{n}} \sum_{j=-k}^{k} P\left(X_{1}>u_{n}, M_{2, r_{n}}^{(1)} \leq u_{n}, Y_{j+1}>v_{n}, M_{j+2, r_{n}}^{(2)} \leq v_{n}\right) \\
& =\lim _{n \rightarrow \infty} \sum_{j=-k}^{k} n P\left(X_{1}>u_{n}, M_{2, r_{n}}^{(1)} \leq u_{n}, Y_{j+1}>v_{n}, M_{j+2, r_{n}}^{(2)} \leq v_{n}\right)
\end{aligned}
$$

By applying again conditions $D^{(s)}\left(u_{n}\right)$ for $\left\{X_{n}\right\}$ and $D^{(t)}\left(v_{n}\right)$ for $\left\{Y_{n}\right\}$, we conclude that the previous limit becomes

$$
\lim _{n \rightarrow \infty} \sum_{j=-k}^{k} n P\left(X_{1}>u_{n}, M_{2, s}^{(1)} \leq u_{n}, Y_{j+1}>v_{n}, M_{j+2, t}^{(2)} \leq v_{n}\right)
$$

For each $\left(\tau_{1}, \tau_{2}\right) \in \mathbb{R}_{+}^{2}$, the value

$$
\xi\left(\tau_{1}, \tau_{2}\right)=\lim _{n \rightarrow \infty} \sum_{j=0}^{2 k} n P\left(X_{k+1}>u_{n} \geq M_{k+2, k+s}^{(1)}, Y_{j+1}>v_{n} \geq M_{j+2, j+t}^{(2)}\right) \geq 0
$$

provided that the limit exists for $\left\{\left(u_{n}, v_{n}\right)\right\}$ such that $n\left(1-F_{X}\left(u_{n}\right)\right) \rightarrow \tau_{1}>0$ and $n\left(1-F_{Y}\left(v_{n}\right)\right) \rightarrow \tau_{2}>0$, as $n \rightarrow \infty$, appears as a quantifying parameter of the asymptotic dependence between $M_{n}^{(1)}$ and $M_{n}^{(2)}$. Once the local dependence conditions are validated, this index depends on the joint behavior of a finite number of the variables of the process. This index contemplates the possibility of joint occurrence of clusters of high values, for each sequence of margins separated by a maximum of $k \geq 0$. By assuming $D^{(s)}\left(u_{n}\right), D^{(t)}\left(v_{n}\right)$ and $D^{(k)}\left(u_{n}, v_{n}\right)$, we do not establish any relation between $s, t$ and $k$, that is, between the minimum distances separating clusters of the same sequence of margins ( $s$ and $t$ ) and the maximum distance between clusters of distinct margins $(k)$. In the following we state two more properties concerning function $\xi\left(\tau_{1}, \tau_{2}\right)$.

Proposition 2.2. Under conditions of Lemma 2.1, if $P\left(M_{n}^{(1)} \leq n / \tau_{1}, M_{n}^{(2)} \leq n / \tau_{2}\right) \rightarrow$ $H\left(\tau_{1}^{-1}, \tau_{2}^{-1}\right)$, as $n \rightarrow \infty$ and $\left(\tau_{1}, \tau_{2}\right) \in \mathbb{R}_{+}^{2}$, for some BEV $\mathrm{d} f H$, then function $\xi\left(\tau_{1}, \tau_{2}\right)$ is homogeneous of order 1 provided it is non constant.

Proof. By Corollary 1.3 in (Chernick, Hsing, and McCormick 1991), we have that $P\left(M_{2, s}^{(1)} \leq\right.$ $\left.u_{n} \mid X_{1}>u_{n}\right) \rightarrow \theta_{X}$, as well as $P\left(M_{2, t}^{(2)} \leq v_{n} \mid Y_{1}>v_{n}\right) \rightarrow \theta_{Y}$, where $\theta_{X}$ and $\theta_{Y}$ are the respective marginal extremal indexes. Now, just observe that

$$
\begin{aligned}
P\left(M_{n}^{(1)} \leq \frac{n}{t \tau_{1}}, M_{n}^{(2)} \leq \frac{n}{t \tau_{2}}\right) \rightarrow & \mathrm{e}^{-\theta_{X} t \tau_{1}} \mathrm{e}^{-\theta_{Y} t \tau_{2}} \mathrm{e}^{\xi\left(t \tau_{1}, t \tau_{2}\right)}=H\left(\left(t \tau_{1}\right)^{-1},\left(t \tau_{2}\right)^{-1}\right) \\
& =H^{t}\left(\tau_{1}^{-1}, \tau_{2}^{-1}\right) \\
& =\left(\mathrm{e}^{-\theta_{X} \tau_{1}} \mathrm{e}^{-\theta_{Y} \tau_{2}} \mathrm{e}^{\xi\left(\tau_{1}, \tau_{2}\right)}\right)^{t}
\end{aligned}
$$

where the second equality is due to a max-stability property of a BEV distribution (Galambos 1987; Theorem 5.2.1). Thus $\xi\left(t \tau_{1}, t \tau_{2}\right)=t \xi\left(\tau_{1}, \tau_{2}\right)$. 
Proposition 2.3. Under conditions of Lemma 2.1, if $\left\{\left(X_{n}, Y_{n}\right)\right\}$ has bivariate extremal index $\theta\left(\tau_{1}, \tau_{2}\right)$, then

$$
\theta\left(\tau_{1}, \tau_{2}\right)=\frac{\theta_{X} \tau_{1}+\theta_{Y} \tau_{2}-\xi\left(\tau_{1}, \tau_{2}\right)}{\tau_{1}+\tau_{2}-\lambda\left(\tau_{1}, \tau_{2}\right)}
$$

where $\lambda\left(\tau_{1}, \tau_{2}\right)=\lim _{n \rightarrow \infty} n P\left(X_{1}>n / \tau_{1}, Y_{1}>n / \tau_{2}\right)$

Proof. Since

$$
\begin{aligned}
\lim _{n \rightarrow \infty} n P\left(\left\{X_{1}>n / \tau_{1}\right\} \cup\left\{Y_{1}>n / \tau_{2}\right\}\right) & =\tau_{1}+\tau_{2}-\lim _{n \rightarrow \infty} n P\left(X_{1}>\frac{n}{\tau_{1}}, Y_{1}>\frac{n}{\tau_{2}}\right) \\
& =\tau_{1}+\tau_{2}-\lambda\left(\tau_{1}, \tau_{2}\right)
\end{aligned}
$$

then

$$
\begin{aligned}
P\left(M_{n}^{(1)} \leq \frac{n}{\tau_{1}}, M_{n}^{(2)} \leq \frac{n}{\tau_{2}}\right) & \rightarrow\left(\mathrm{e}^{-\theta_{X} \tau_{1}} \mathrm{e}^{-\theta_{Y} \tau_{2}} \mathrm{e}^{\xi\left(\tau_{1}, \tau_{2}\right)}\right) \\
& =\exp \left\{-\theta\left(\tau_{1}, \tau_{2}\right)\left(\tau_{1}+\tau_{2}-\lambda\left(\tau_{1}, \tau_{2}\right)\right)\right\}
\end{aligned}
$$

with $\theta\left(\tau_{1}, \tau_{2}\right)$ satisfying Equation (9).

Observe that $\lambda\left(\tau_{1}, \tau_{2}\right)$ above corresponds to the bivariate upper tail copula function considered in Schmidt and Stadtmüller (2006). See also Li (2009) and references therein. The bivariate extremal index was introduced in Nandagopalan (1994). More recent developments can be seen in Pereira, Martins, and Ferreira (2017).

If the marginals of the limiting BEV $H$ are independent, we have $\xi\left(\tau_{1}, \tau_{2}\right)=0$. However, a residual tail dependence measured through the rate of convergence of $\xi\left(\tau_{1}, \tau_{2}\right)$ towards zero may occur. This type of dependence is usually ruled in the literature through the Ledford and Tawn coefficient $\eta$, defined in Equation (1). This is addressed in the next section.

\section{Pre-asymptotic dependence between $M_{n}^{(1)}$ and $M_{n}^{(2)}$}

We are going to analyze the asymptotic dependence function $\xi\left(\tau_{1}, \tau_{2}\right)$ in Equation (8), by considering two particular cases for $s$ and $t$ often addressed in the literature.

Proposition 3.1. Under conditions of Lemma 2.1, if $s=t=1$ and, as $n \rightarrow \infty$,

$$
n P\left(X_{i}>u_{n}, Y_{j}>v_{n}\right) \sim n^{-\left(1 / \eta_{i j}-1\right)} \mathcal{L}_{\eta_{i j}}\left(\frac{n}{\tau_{1}}, \frac{n}{\tau_{2}}\right)
$$

holds for all $j=1, \ldots, 2 k+1$ and $i=k+1$, with $\eta_{i j} \equiv \eta_{i j}\left(\tau_{1}, \tau_{2}\right) \in(0,1]$ and $\mathcal{L}_{\eta_{i j}}$ slowly varying functions, then

$$
\xi\left(\tau_{1}, \tau_{2}\right) \sim n^{-(1 / \eta-1)} \mathcal{L}^{*}\left(\frac{n}{\tau_{1}}, \frac{n}{\tau_{2}}\right)
$$

where $\eta=\max \left\{\eta_{i j}: j=1, \ldots, 2 k+1, i=k+1\right\}$ and

$$
\mathcal{L}^{*}\left(\frac{n}{\tau_{1}}, \frac{n}{\tau_{2}}\right)=\sum_{j=0}^{2 k} n^{-\left(1 / \eta_{i j}-1 / \eta\right)} \mathcal{L}_{\eta_{i j}}\left(\frac{n}{\tau_{1}}, \frac{n}{\tau_{2}}\right)
$$

is a slowly varying function. 
Proof. Under conditions $D^{(1)}\left(u_{n}\right)$ and $D^{(1)}\left(v_{n}\right)$, we have $\theta_{X}=\theta_{Y}=1$ (Chernick, Hsing, and McCormick 1991; Corollary 1.3). Now observe that,

$$
\lim _{n \rightarrow \infty} P\left(M_{n}^{(1)} \leq u_{n}, M_{n}^{(2)} \leq v_{n}\right)=\mathrm{e}^{-v_{1}} \mathrm{e}^{-v_{2}} \mathrm{e}^{\xi\left(\tau_{1}, \tau_{2}\right)}
$$

with $v_{1}=\tau_{1}, v_{2}=\tau_{2}$ and

$$
\xi\left(\tau_{1}, \tau_{2}\right)=\lim _{n \rightarrow \infty} \sum_{j=0}^{2 k} n P\left(X_{k+1}>u_{n}, Y_{j+1}>v_{n}\right)
$$

for all $k \geq 0$.

In the context of Proposition 3.1 we have $\xi$-asymptotic tail independence if $\eta<1$ or if $\eta=1$ and $\mathcal{L}^{*}\left(\frac{n}{\tau_{1}}, \frac{n}{\tau_{2}}\right) \rightarrow 0$, as $n \rightarrow \infty$ (which holds if $\mathcal{L}_{\eta_{i j}}\left(\frac{n}{\tau_{1}}, \frac{n}{\tau_{2}}\right) \rightarrow 0$, for all $j=1, \ldots, 2 k+1$, $i=k+1$, such that $\left.\eta_{i j}=1\right)$. This case lead us to $\xi\left(\tau_{1}, \tau_{2}\right)=0$.

We have $\xi$-tail dependence if $\eta=1$ and $\mathcal{L}^{*}\left(\frac{n}{\tau_{1}}, \frac{n}{\tau_{2}}\right) \rightarrow c>0$, as $n \rightarrow \infty$ (which holds if $\mathcal{L}_{\eta_{i j}}\left(\frac{n}{\tau_{1}}, \frac{n}{\tau_{2}}\right) \rightarrow c_{j}>0$, for some $j=1, \ldots, 2 k+1, i=k+1$, such that $\left.\eta_{i j}=1\right)$. Now we obtain $\xi\left(\tau_{1}, \tau_{2}\right)>0$.

Observe that, in order to have $\xi\left(\tau_{1}, \tau_{2}\right)=0$, all random pairs $\left(X_{i}, Y_{j}\right), j=1, \ldots, 2 k+1$, $i=k+1$, must be asymptotic tail independent. On the other hand, if one random pair is tail dependent then $\xi\left(\tau_{1}, \tau_{2}\right)>0$. Notice also that this evaluation is based on exceedances of high thresholds. In the next case our analysis is based on down-crossings of extreme thresholds.

Proposition 3.2. Under conditions of Lemma 2.1, if $s=t=2$ and

$$
n P\left(X_{i} \geq u_{n}>X_{i+1}, Y_{j} \geq v_{n}>Y_{j+1}\right) \sim n^{-\left(1 / \beta_{i j}-1\right)} \mathcal{L}_{\beta_{i j}}\left(\frac{n}{\tau_{1}}, \frac{n}{\tau_{2}}\right)
$$

holds, as $n \rightarrow \infty$, for all $j=1, \ldots, 2 k+1$ and $i=k+1$, with $\beta_{i j} \equiv \beta_{i j}\left(\tau_{1}, \tau_{2}\right) \in(0,1]$ and $\mathcal{L}_{\beta_{i j}}$ slowly varying functions. Then

$$
\xi\left(\tau_{1}, \tau_{2}\right) \sim n^{-(1 / \beta-1)} \mathcal{L}^{* *}\left(\frac{n}{\tau_{1}}, \frac{n}{\tau_{2}}\right)
$$

where $\beta=\max \left\{\beta_{i j}: j=1, \ldots, 2 k+1, i=k+1\right\}$ and

$$
\mathcal{L}^{* *}\left(\frac{n}{\tau_{1}}, \frac{n}{\tau_{2}}\right)=\sum_{j=0}^{2 k} n^{-\left(1 / \beta_{i j}-1 / \beta\right)} \mathcal{L}_{\beta_{i j}}\left(\frac{n}{\tau_{1}}, \frac{n}{\tau_{2}}\right)
$$

is a slowly varying function. Moreover if we assume, as $n \rightarrow \infty$, that

$$
n P\left(\bigcap_{i \in I}\left\{X_{i}>u_{n}\right\}, \bigcap_{j \in J}\left\{Y_{j}>v_{n}\right\}\right) \sim n^{-\left(1 / \eta_{I, J}-1\right)} \mathcal{L}_{\eta_{I, J}}\left(\frac{n}{\tau_{1}}, \frac{n}{\tau_{2}}\right)
$$


for all $I \subseteq\{k+1, k+2\}$ and $J \subseteq\{1, \ldots, 2 k+2\}$, then $\beta=\max \left\{\eta_{i j}: j=1, \ldots, 2 k+1, i=k+1\right\}$ and

$$
\begin{aligned}
\mathcal{L}_{\beta_{i j}}\left(\frac{n}{v_{1}}, \frac{n}{v_{2}}\right) \sim & \mathcal{L}_{\eta_{i j}}\left(\frac{n}{\tau_{1}}, \frac{n}{\tau_{2}}\right)-n^{-\left(1 / \eta_{\{i\},\{j, j+1\}}-1\right)} \mathcal{L}_{\eta_{\{i\},\{j, j+1\}}}\left(\frac{n}{\tau_{1}}, \frac{n}{\tau_{2}}\right) \\
& -n^{-\left(1 / \eta_{\{i, i+1\},\{j\}}-1\right)} \mathcal{L}_{\eta_{\{i, i+1\},\{\}\}}}\left(\frac{n}{\tau_{1}}, \frac{n}{\tau_{2}}\right) \\
& +n^{-\left(1 / \eta_{\{i, i+1\},\{j, j+1\}}-1\right)} \mathcal{L}_{\eta_{\{i, i+1\},\{j, j+1\}}}\left(\frac{n}{\tau_{1}}, \frac{n}{\tau_{2}}\right)
\end{aligned}
$$

where $\eta_{i j} \equiv \eta_{\{i\},\{j\}}$.

Proof. Just notice that Equation (12) holds with $v_{1}=\tau_{1} \theta_{1}, \nu_{2}=\tau_{2} \theta_{2}, \theta_{1}, \theta_{2} \in(0,1]$ and

$$
\xi\left(\tau_{1}, \tau_{2}\right)=\lim _{n \rightarrow \infty} \sum_{j=0}^{2 k} n P\left(X_{k+1} \geq u_{n}>X_{k+2}, Y_{j+1} \geq v_{n}>Y_{j+2}\right)
$$

for all $k \geq 0$.

The second part is straightforward from Proposition 2 of Ferreira and Ferreira (2012).

Observe that $\beta_{i j}$ is similar to the up-crossings asymptotic tail independent coefficient introduced in Ferreira and Ferreira (2012). Analogously to the previous case, we can exploit tail (in)dependence under the point of view of down-crossings of high levels. Therefore, we have $\xi$-asymptotic tail independence if $\beta<1$ or if $\beta=1$ and $\mathcal{L}^{* *}\left(\frac{n}{\tau_{1}}, \frac{n}{\tau_{2}}\right) \rightarrow 0$, as $n \rightarrow \infty$ (leading to $\xi\left(\tau_{1}, \tau_{2}\right)=0$ ) and $\xi$-tail dependence if $\beta=1$ and $\mathcal{L}^{* *}\left(\frac{n}{\tau_{1}}, \frac{n}{\tau_{2}}\right) \rightarrow c>0$, as $n \rightarrow \infty$ (obtaining $\xi\left(\tau_{1}, \tau_{2}\right)>0$ ). Once again, in order to have $\xi\left(\tau_{1}, \tau_{2}\right)=0$, all random pairs $\left(X_{i}, Y_{j}\right), j=1, \ldots, 2 k+1, i=k+1$, must be down-crossings asymptotic tail independent, but if one random pair is down-crossings tail dependent then $\xi\left(\tau_{1}, \tau_{2}\right)>0$.

Example 3.1. Let $\left\{X_{n}^{*}\right\}$ and $\left\{Y_{n}^{*}\right\}$ be stationary sequences such that conditions $D^{(s)}\left(u_{n}\right)$ and $D^{(t)}\left(v_{n}\right)$ respectively hold, and $\left\{Z_{n}\right\}$ be an i.i.d. sequence independent of $\left\{\left(X_{n}^{*}, Y_{n}^{*}\right)\right\}$, all having common margin standard Fréchet. Consider

$$
X_{n}=X_{n}^{*} \vee Z_{n}^{1 / \alpha} \text { and } Y_{n}=Y_{n}^{*} \vee Z_{n}^{1 / \rho}
$$

where $\alpha, \rho \in(0,1)$, corresponding to a pMAX model introduced in Ferreira and Ferreira (2014). We have that $\left\{X_{n}\right\}$ and $\left\{Y_{n}\right\}$ also satisfy conditions $D^{(s)}\left(u_{n}\right)$ and $D^{(t)}\left(v_{n}\right)$, respectively. Consider the particular case where $\left\{Y_{n}^{*}=X_{n}^{*} \mathbb{1}_{\left\{J_{n}=0\right\}}+X_{n+1}^{*} \mathbb{1}_{\left\{J_{n}=1\right\}}\right\}$, with $\left\{J_{n}\right\}$ an i.i.d. Bernoulli sequence and $s=t=1$. We have $\theta_{X}=\theta_{X^{*}}=1, \theta_{Y}=\theta_{Y^{*}}=1$ (see Proposition 2.2 in Ferreira and Ferreira 2014) and $\xi\left(\tau_{1}, \tau_{2}\right)$ is given by Equation (13). Assuming that, as $n \rightarrow \infty$,

$$
n P\left(X_{i}^{*}>u_{n}, X_{l}^{*}>v_{n}\right) \sim n^{-\left(1 / \eta_{i, l}^{\left(X^{*}\right)}-1\right)} \mathcal{L}_{\eta_{i, l}^{\left(X^{*}\right)}}\left(\frac{n}{\tau_{1}}, \frac{n}{\tau_{2}}\right)
$$

for $i=k+1$ and $l=1, \ldots, 2 k+2$, thus

$$
\begin{aligned}
n P\left(X_{i}^{*}>u_{n}, Y_{j}^{*}>v_{n}\right) & =n P\left(X_{i}^{*}>u_{n}, X_{j}^{*}>v_{n}\right)(1-p)+n P\left(X_{i}^{*}>u_{n}, X_{j+1}^{*}>v_{n}\right) p \\
& \sim n^{-\left(1 / \eta_{i, j}^{\left(X^{*}\right)}-1\right)} \mathcal{L}_{\eta_{i, j}^{\left(X^{*}\right)}}\left(\frac{n}{\tau_{1}}, \frac{n}{\tau_{2}}\right)+n^{-\left(1 / \eta_{i, j+1}^{\left(X^{*}\right)}-1\right)} \mathcal{L}_{\eta_{i, j+1}^{\left(X^{*}\right)}}\left(\frac{n}{\tau_{1}}, \frac{n}{\tau_{2}}\right) \\
& \sim n^{-\left(1 / \eta_{i, j}^{\left(X^{*}, Y^{*}\right)}-1\right)} \mathcal{L}_{\eta_{i, j}^{\left(X^{*}, Y^{*}\right)}}\left(\frac{n}{\tau_{1}}, \frac{n}{\tau_{2}}\right)
\end{aligned}
$$


where, for $i=k+1$ and $j=1, \ldots, 2 k+1, \eta_{i, j}^{\left(X^{*}, Y^{*}\right)}=\max \left\{\eta_{i, j}^{\left(X^{*}\right)}, \eta_{i, j+1}^{\left(X^{*}\right)}\right\}=1$, since $\eta_{k+1, k+1}^{\left(X^{*}\right)}=$ 1 and thus $\eta_{k+1, k+1}^{\left(X^{*}, Y^{*}\right)}=1$.

Therefore, by applying Proposition 2.6 in Ferreira and Ferreira (2014), we have that Equation (11) holds with

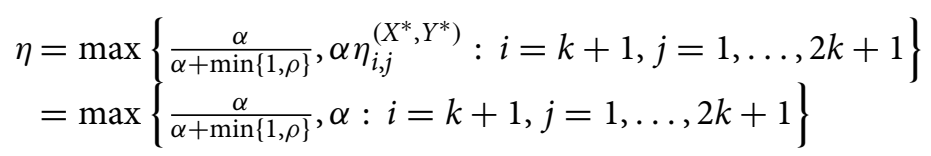

Example 3.2. Consider again the pMAX model above in Equation (20), where $\alpha, \rho \in[1, \infty)$. Consider the particular case where $k=1$, and $s=t=2,\left\{X_{n}^{*}\right\} 1$-dependent (and thus satisfy $\left.D^{(2)}\left(u_{n}\right)\right)$ and $\left\{Y_{n}^{*}=X_{n+3}^{*} \mathbb{1}_{\left\{J_{n}=0\right\}}+X_{n+4}^{*} \mathbb{1}_{\left\{J_{n}=1\right\}}\right\}$, with $\left\{J_{n}\right\}$ an i.i.d. Bernoulli sequence. We have $v_{1}=\theta_{X}=\theta_{X^{*}}, v_{2}=\theta_{Y}=\theta_{Y^{*}}$ (see Proposition 2.2 in Ferreira and Ferreira 2014) and

$$
\begin{gathered}
\xi\left(\tau_{1}, \tau_{2}\right)=n P\left(X_{2}>u_{n} \geq X_{3}, Y_{1}>v_{n} \geq Y_{2}\right)+n P\left(X_{2}>u_{n} \geq X_{3}, Y_{2}>v_{n} \geq Y_{3}\right) \\
+n P\left(X_{2}>u_{n} \geq X_{3}, Y_{3}>v_{n} \geq Y_{4}\right)
\end{gathered}
$$

Since $\left\{X_{n}^{*}\right\}$ 1-dependent, as $n \rightarrow \infty$, we have

$$
n P\left(X_{2}^{*}>u_{n}, X_{j}^{*}>v_{n}\right) \sim \frac{\tau_{1} \tau_{2}}{n}
$$

for $j \geq 4$, and thus $\eta_{2, j}^{\left(X^{*}, Y^{*}\right)}=1 / 2$.

By Proposition 2.6 in Ferreira and Ferreira (2014), we have that Equation (15) holds with

$$
\beta=\max \left\{\frac{1}{\alpha+1}, \frac{1}{\rho+1}, \frac{1}{2}\right\}
$$

The example below addresses factor models, used in the modeling of large losses within, e.g., insurance (Lescourret and Robert 2006) and finance (Ferreira and Canto e Castro 2010; Ferreira and Ferreira 2015). See also Li (2009) and references therein.

Example 3.3. Consider the mixture model, $\left(X_{n}, Y_{n}\right)=\left(R X_{n}^{*}, R Y_{n}^{*}\right)$, where sequences $\left\{X_{n}^{*}\right\}$ and $\left\{Y_{n}^{*}\right\}$ satisfy, respectively, conditions $D^{(s)}\left(u_{n}\right)$ and $D^{(t)}\left(v_{n}\right)$ and have extremal indexes $\theta_{X^{*}}$ and $\theta_{Y^{*}}$, and where $R$ is a positive r.v. independent of $\left\{\left(X_{n}^{*}, Y_{n}^{*}\right)\right\}$ and such that $E(R)<\infty$. If $\left\{\left(X_{n}^{*}, Y_{n}^{*}\right)\right\}$ satisfies $D^{(k)}\left(u_{n}, v_{n}\right)$ then $\left\{\left(X_{n}, Y_{n}\right)\right\}$ satisfies it as well. Let $u_{n}^{*}=n / \tau_{1}^{*}$ and $v_{n}^{*}=$ $n / \tau_{2}^{*}$ be normalized levels for $\left\{X_{n}^{*}\right\}$ and $\left\{Y_{n}^{*}\right\}$. Thus, they are normalized levels for $\left\{X_{n}\right\}$ and $\left\{Y_{n}\right\}$ with $\tau_{1}=E(R) \tau_{1}^{*}$ and $\tau_{2}=E(R) \tau_{2}^{*}$, respectively. By applying Equation (8), we have

$$
\begin{aligned}
\xi\left(\tau_{1}, \tau_{2}\right) & =\lim _{n \rightarrow \infty} \int_{0}^{\infty} \sum_{j=0}^{2 k} n P\left(X_{k+1}^{*}>\frac{n}{\tau_{1}^{*} r} \geq M_{k+2, k+s}^{(1)}, Y_{j+1}^{*}>\frac{n}{\tau_{2}^{*} r} \geq M_{j+2, j+t}^{(2)}\right) \mathrm{d} F_{R}(r) \\
& =\lim _{n \rightarrow \infty} \int_{0}^{\infty} \xi^{*}\left(\tau_{1}^{*} r, \tau_{2}^{*} r\right) \mathrm{d} F_{R}(r)=\xi^{*}\left(\tau_{1}^{*}, \tau_{2}^{*}\right) E(R)
\end{aligned}
$$

if $\xi^{*}\left(\tau_{1}^{*}, \tau_{2}^{*}\right)$ exists and is homogeneous of order 1 . Assuming that, as $n \rightarrow \infty$,

$$
n P\left(X_{i}^{*}>\frac{n}{\tau_{1}^{*}}, Y_{j}^{*}>\frac{n}{\tau_{2}^{*}}\right) \sim n^{-\left(1 / \eta_{i j}^{*}-1\right)} \mathcal{L}_{\eta_{i j}^{*}}\left(\frac{n}{\tau_{1}^{*}}, \frac{n}{\tau_{2}^{*}}\right)
$$


we have, by applying the dominated convergence theorem,

$$
\begin{aligned}
n P\left(R X_{i}^{*}>\frac{n}{\tau_{1}^{*}}, R Y_{j}^{*}>\frac{n}{\tau_{2}^{*}}\right) & =\int_{0}^{\infty} n P\left(X_{i}^{*}>\frac{n}{\tau_{1}^{*} r}, Y_{j}^{*}>\frac{n}{\tau_{2}^{*} r}\right) \mathrm{d} F_{R}(r) \\
& \sim \int_{0}^{\infty} r^{1 / \eta_{i j}^{*} n^{-\left(1 / \eta_{i j}^{*}-1\right)}} \mathcal{L}_{\eta_{i j}^{*}}\left(\frac{n}{\tau_{1}^{*} r}, \frac{n}{\tau_{2}^{*} r}\right) \mathrm{d} F_{R}(r) \\
& \sim \int_{0}^{\infty} r^{1 / \eta_{i j}^{*} n^{-\left(1 / \eta_{i j}^{*}-1\right)}} \mathcal{L}_{\eta_{i j}^{*}}\left(\frac{n}{\tau_{1}^{*}}, \frac{n}{\tau_{2}^{*}}\right) \mathrm{d} F_{R}(r) \\
& =n^{-\left(1 / \eta_{i j}^{*}-1\right)} \mathcal{L}_{\eta_{i j}^{*}}\left(\frac{n}{\tau_{1}^{*}}, \frac{n}{\tau_{2}^{*}}\right) E\left(R^{1 / \eta_{i j}^{*}}\right)
\end{aligned}
$$

provided $E\left(R^{1 / \eta_{i j}^{*}}\right)$ exists. Thus, we can state

$$
n P\left(X_{i}>\frac{n}{\tau_{1}}, Y_{j}>\frac{n}{\tau_{2}}\right) \sim n^{-\left(1 / \eta_{i j}-1\right)} \mathcal{L}_{\eta_{i j}}\left(\frac{n}{\tau_{1}}, \frac{n}{\tau_{2}}\right)
$$

where $\eta_{i j}=\eta_{i j}^{*}$ and $\mathcal{L}_{\eta_{i j}}\left(\frac{n}{\tau_{1}}, \frac{n}{\tau_{2}}\right)=\mathcal{L}_{\eta_{i j}^{*}}\left(\frac{n}{\tau_{1}^{*}}, \frac{n}{\tau_{2}^{*}}\right) E\left(R^{1 / \eta_{i j}^{*}}\right)$.

\section{Discussion}

In this paper we introduce a new index, $\xi\left(\tau_{1}, \tau_{2}\right)$, in order to measure a (pre-)asymptotic dependence between the component-wise maxima of a bivariate stationary sequence. We consider the marginal local behavior of the sequence ruled through Chernick, Hsing, and McCormick (1991) dependence conditions, $D^{(s)}\left(u_{n}\right)$ and $D^{(t)}\left(v_{n}\right)$, for some $s, t>0$, along with a bivariate local dependence condition $D^{(k)}\left(u_{n}, v_{n}\right), k>0$, defined here. An empirical approach to validate some $D^{(s)}\left(u_{n}\right)$ was presented in Ferreira and Ferreira (2018). See also Süveges (2007). An automated statistical method for joint selection of threshold $u_{n}$ and parameter $s$ can be seen in Fukutome, Liniger, and Süveges (2014). We believe that both methodologies can be extended to $D^{(k)}\left(u_{n}, v_{n}\right)$, at least through condition Equation (7). In Ledford and Tawn (1997) we can find parametric estimation based on maximum likelihood (and thus not suitable in our context which assumes dependence between random pairs), as well as, a non parametric proposal. This approach will be a starting point to address this topic in a future work.

\section{Acknowledgments}

The authors would like to thank the reviewers for their valuable comments that contributed to improve this article. The first author's research was partially supported by the research unit UID/MAT/00212/2013. The second author was financed by Portuguese Funds through FCT-Fundação para a Ciência e a Tecnologia within the Projects UID/MAT/00013/2013, UID/MAT/00006/2013 and by the research center CEMAT (Instituto Superior Técnico, Universidade de Lisboa) through the Project UID/Multi/04621/2013.

\section{References}

Bortot, P., and J. A. Tawn. 1998. Models for the extremes of Markov chains. Biometrika 85 (4):851-67. 
Chernick, M. R., T. Hsing, and W. P. McCormick. 1991. Calculating the extremal index for a class of stationary sequences. Advance in Applied Probability 23:835-850.

Davis, R. A. 1982. Limit laws for the maximum and minimum of stationary sequences. Zeitschrift fr Wahrscheinlichkeitstheorie und Verwante Gebiete 61:31-42.

Ferreira, H., and M. Ferreira. 2014. Extremal behavior of pMAX processes. Statistics and Probability Letters 93:46-57.

Ferreira, H., and M. Ferreira. 2015. Extremes of scale mixtures of multivariate time series. Journal of Multivariate Analysis 137:82-99.

Ferreira, H., and M. Ferreira. 2018. Estimating the extremal index through local dependence. Annales de l'Institut Henri Poincarè, Probabilities et Statistiques 54:587-605.

Ferreira, M., and H. Ferreira. 2012. On extremal dependence: Some Contributions. Test 21:566-83.

Ferreira, M., and L. Canto e Castro. 2010. Modeling rare events through a pRARMAX process. Journal of Statistical Planning and Inference 140 (11):3552-66.

Fukutome, S., M. A. Liniger, and M. Süveges. 2014. Automatic threshold and run parameter selection: A climatology for extreme hourly precipitation in Switzerland. Theoretical and Applied Climatology 120 (3): 403-16.

Galambos, J. 1987. The asymptotic theory of extreme order statistics. 2nd ed. Melbourne, Krieger.

Hsing, T. 1989. Extreme value theory for multivariate stationary sequences. Journal of Multivariate Analysis 29:274-91.

Leadbetter, M. R., G. Lindgren, and H. Rootzén. 1983. Extremes and related properties of random sequences and processes. Berlin, Springer.

Leadbetter, M. R., and S. Nandagopalan. 1989. On exceedance point processes for stationary sequences under mild oscillation restrictions. Lecture Notes in Statistics 51:69-80.

Ledford, A. W., and J. A. Tawn. 1996. Statistics for near independence in multivariate extreme values. Biometrika 83:169-87.

Ledford, A. W., and J. A. Tawn. 1997. Modelling dependence within joint tail regions. Journal of the Royal Statistical Society 59:475-99.

Lescourret, L., and C. Robert. 2006. Extreme dependence of multivariate catastrophic losses. Scandinavian Actuarial Journal 2006 (4): 203-25.

Li, H. 2009. Orthant tail dependence of multivariate extreme value distributions. Journal of Multivariate Analysis 100 (1): 243-56.

Nandagopalan, S. 1994. On the multivariate extremal index. Journal of Research, National Institute of Standards and Technology 99:543-50.

Pereira, L., A. P. Martins, and H. Ferreira. 2017. Clustering of high values in random fields. Extremes 20:807-38.

Poon, S.-H., M. Rockinger, and J. A. Tawn. 2003. Modelling extreme-value dependence in international stock markets. Statistica Sinica 13:929-53.

Ramos, A., and A. Ledford. 2011. Alternative point process framework for modeling multivariate extreme values. Communications in Statistics - Theory and Methods 40:2205-24.

Schmidt, R., and U. Stadtmüller. 2006. Nonparametric estimation of tail dependence. Scandinavian Journal of Statistics 33:307-35.

Smith, R. L. 1990. Max-stable processes and spatial extremes. Pre-print. Chapel Hill, NC: University of North Carolina.

Süveges, M. 2007. Likelihood estimation of the extremal index. Extremes 10:41-55.

Tiago de Oliveira, J. 1962/1963. Structure theory of bivariate extremes extensions. Estudes de Mathematica, Estatistica e Econometrica 7:165-195. 